\title{
Toward quantitative Kelvin probe force microscopy of nanoscale potential distributions
}

\author{
Robert Baier, ${ }^{1, *}$ Caspar Leendertz, ${ }^{1}$ Martha Ch. Lux-Steiner, ${ }^{1}$ and Sascha Sadewasser ${ }^{1,2}$ \\ ${ }^{1}$ Helmholtz-Zentrum Berlin für Materialien und Energie, Hahn-Meitner-Platz 1, 14109 Berlin, Germany \\ ${ }^{2}$ International Iberian Nanotechnology Laboratory, Avenida Mestre José Veiga s/n, 4715-330 Braga, Portugal \\ (Received 20 December 2011; revised manuscript received 15 February 2012; published 19 April 2012)
}

\begin{abstract}
Kelvin probe force spectroscopy (KPFS) and finite-element method (FEM) simulations were employed to investigate the averaging effect of the work function signals of nanoscale potential distributions in Kelvin probe force microscopy (KPFM). A KPFS routine is presented that enables meaningful experimental results even for electronically inhomogeneous KPFM tips. By use of this routine a strong distance dependence of the averaging effect is revealed. A combination of KPFS experiments and FEM simulations is applied to quantify the averaging effect, which simplifies comparison among different experiments and to theory. No influence of surface topography on the averaging effect was observed.
\end{abstract}

DOI: 10.1103/PhysRevB.85.165436

PACS number(s): 68.37.-d, 73.22.-f

\section{INTRODUCTION}

All atomic force microscopy ${ }^{1}$ (AFM) methods use a tip at the end of a cantilever as sensor to obtain spatially resolved information about the properties of a surface. Consequently, the maximum lateral resolution in such measurements is mainly determined by the dimension of that segment of the tip which contributes most significantly to the total interaction between tip and sample. For example, to achieve true atomic resolution in noncontact AFM it is necessary that the foremost atom of the tip-apex contributes significantly to the total force gradient. ${ }^{2}$ If a larger tip segment contributes strongly to the imaging signal, the spatial information from individual surface features is averaged out and the resolution decreased.

Kelvin probe force microscopy ${ }^{3,4}$ (KPFM) is based on AFM and represents a very powerful tool to obtain laterally resolved images of a sample's surface potential or charge distribution. $^{4-6}$ Its popularity in nanoscience is currently strongly increasing, finding application in a broad variety of material systems such as semiconductors, ${ }^{7-9}$ insulators, ${ }^{10-12}$ and organic molecules. ${ }^{13-15}$ Recently, potential variations on the atomic scale have also been observed experimentally ${ }^{16-18}$ as well as theoretically. ${ }^{19,20}$

Despite this strongly growing interest in KPFM and the continuing pursuit of subnanometer scales, the quantification and comparison of work function changes on a nanometer scale is still a critical issue. The comparably large tip-sample distances in many cantilever-based KPFM experiments (several nanometers) in combination with the long-range nature of the electrostatic force result in a rather large tip segment contributing to the KPFM imaging signal. ${ }^{21-23}$ Therefore, the work function signals of nanoscale potential distributions (NPDs) in KPFM are also subject to an averaging effect. Even if the tip is located directly above the center of a nanoscale potential distribution, the KPFM imaging signal is not solely obtained from the NPD, but also from the adjacent surface area. The averaging effect causes a decrease of the NPD work function signal detected in KPFM in dependence on the tip-sample distance. This basically prohibits any direct quantitative comparison of results from different experiments or to theory.

The averaging effect in KPFM has previously been addressed by several authors. Modeling the electrostatic interaction between tip and sample with the help of a capacitor approach, Jacobs et $a .^{24}$ revealed that the KPFM imaging signal represents a weighted average of the sample's surface potential and demonstrated its effects on KPFM measurements at potential steps and NPDs. A similar approach was utilized by Sadewasser et al. ${ }^{25}$ Palleau et al. ${ }^{26}$ investigated the averaging effect by means of numerical simulations and experiments to estimate the surface charge density of polymethylmethacrylate thin films. Belaidi et al ${ }^{27}$ applied finite-element method (FEM) simulations to analyze the resolution limits at potential steps, and Leendertz et al. ${ }^{28}$ at NPDs. Zerweck et $a .^{29}$ pointed out differences in the averaging effect between the AM and FM modes of KPFM.

In this paper we reconsider the averaging effect of the KPFM work function signal. We analyze the influence of different tip-sample distances as well as the impact of surface topography for KPFM measurements at NPDs. We find a strong distance dependence of the averaging effect, while surface topography plays only an insignificant role. By applying Kelvin probe force spectroscopy ${ }^{6,18}$ (KPFS) in experiment and simulation, we were able to quantify the averaging effect. This experimental procedure simplifies comparison among different experiments and to theory.

\section{EXPERIMENT}

All measurements were performed in an ultrahigh-vacuum (UHV) KPFM setup ${ }^{30,31}$ (base pressure $<10^{-10}$ mbar) using the amplitude modulation technique at the second resonance frequency of the cantilever for detection of the contact potential difference (CPD). The applied ac voltage was $100 \mathrm{mV}$. For simultaneous topography measurements the conventional frequency modulation technique at the first cantilever resonance $\left(f_{0} \approx 75 \mathrm{kHz}\right)$ was utilized. The measurements were performed with cantilevers with Pt-Ir-coated Si tips. ${ }^{32}$ Absolute work function $(\Phi)$ values were obtained by calibration measurements on highly oriented pyrolytic graphite.

The KPFS experiments were conducted as follows: four KPFS spectra (1024 points/spectrum) were taken directly at the feature NPD of interest, starting at a minimum tip-sample distance $z_{\min }$ of about $2 \mathrm{~nm}$. The sweep distance was $500 \mathrm{~nm}$. The four spectra were averaged to reduce the noise level of 

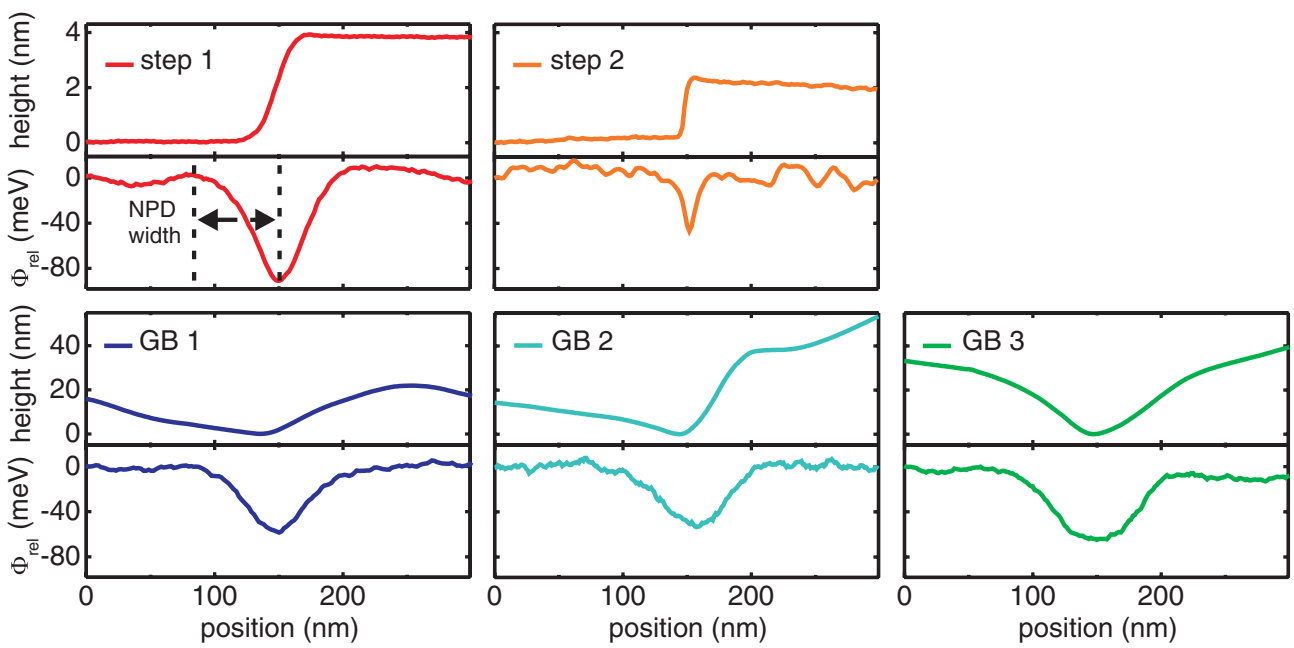

FIG. 1. (Color online) KPFM line profiles of topography and relative work function $\left(\Phi_{\text {rel }}\right)$ across the various NPDs from Table I, obtained at the minimum tip-sample distances stated in Table I. All work function line profiles were shifted in such a way that $\Phi_{\text {rel }}$ outside the NPD is at 0 .

the signal. The same procedure was repeated at a position 100-300 nm laterally away from the NPD (we refer to this as "background" in the following). In order to obtain the pure distance dependence of the NPD work function signal $(\Delta \Phi)$, the two spectra were subtracted one from the other to create the so-called KPFS difference spectrum. This procedure avoids the influence of electronic inhomogeneities of the tip on the experimental results, as demonstrated in Sec. III B. For further signal improvement a 20-point adjacent average of the difference spectrum was generated. The minimum tip-sample distance $z_{\min }$ was calibrated to absolute values by distance spectroscopy. The zero point was determined by the onset of a strong damping signal, indicating short-range atomic forces between tip and sample. To account for the oscillation of the cantilever during the KPFS measurements (an oscillation amplitude of $A=10 \mathrm{~nm}$ was used for all experiments) an effective tip-sample distance (from now on labeled as " $z$ ") was calculated from the minimum tip-sample distance $z_{\min }$. It was shown before ${ }^{33,34}$ that an effective tip-sample distance can be used in good approximation to account for the cantilever oscillation in KPFM. ${ }^{35}$

A true three-dimensional (3D) FEM simulation was employed to simulate KPFS measurements. ${ }^{28,34}$ In the simulation the electrostatic potential distribution was calculated for the 3D geometry shown in inset 2 of Fig. 3. By minimizing the electrostatic attraction between tip and sample the CPD was calculated. The NPD was defined as a potential dip with the shape of a space-charge region (SCR). Two spectra, directly at the NPD and in the background, were subtracted one from the other in order to obtain the pure distance dependence of the NPD (the same procedure as for the experimental data). A cantilever oscillation is indirectly considered by comparing the tip-sample distance of the simulations to an effective tip-sample distance in the experiments.

Experiments were performed on UHV-cleaved $p$-type $\mathrm{GaAs}(110)$ and on polycrystalline $\mathrm{CuIn}_{0.7} \mathrm{Ga}_{0.3} \mathrm{Se}_{2}$ (CIGSe) thin films. The GaAs(110) surface exposes several steps, due to cleavage in UHV. At the step edges positive charges accumulate and noticeably lower the work function. ${ }^{7}$ Grain boundaries (GBs) of CIGSe, on the other hand, frequently show different electronic properties from the bulk of the material, presumably due to the presence of localized charged surface states. ${ }^{36,37}$ The frequent appearance of NPDs in both materials makes them ideal model systems for our studies.

In total, five different NPDs were investigated, two NPDs at step edges of differently doped $p$-type GaAs(110) (from

TABLE I. Overview of various properties of the different NPDs investigated within this paper. Both the measured NPD work function signal $\left(\Delta \Phi=\Phi_{\mathrm{NPD}}-\Phi_{\text {background }}\right)$ and the NPD width were determined from Fig. 1 . The absolute $\Delta \Phi$ are extracted by comparison to simulations, as described below.

\begin{tabular}{|c|c|c|c|c|c|}
\hline Label & Step 1 & Step 2 & GB1 & GB2 & GB3 \\
\hline Material & $\operatorname{GaAs}(110)$ & GAs(110) & CIGSe & CIGSe & CIGSe \\
\hline Type & $p$ type & $p$ type & $p$ type & $p$ type & $p$ type \\
\hline Topography & Steplike & Steplike & Valleylike & Valleylike & Valleylike \\
\hline NPD width (nm) & $65 \pm 5$ & $20 \pm 5$ & $65 \pm 5$ & $60 \pm 5$ & $65 \pm 5$ \\
\hline$z_{\min }(\mathrm{nm})$ & $2.7 \pm 0.1$ & $0.9 \pm 0.1$ & $2.2 \pm 0.1$ & $2.2 \pm 0.1$ & $4.7 \pm 0.1$ \\
\hline$z(\mathrm{~nm})$ & $7.7 \pm 0.1$ & $5.9 \pm 0.1$ & $7.2 \pm 0.1$ & $7.2 \pm 0.1$ & $9.7 \pm 0.1$ \\
\hline Measured $\Delta \Phi(\mathrm{meV})$ & $-90 \pm 5$ & $-47 \pm 5$ & $-58 \pm 5$ & $-53 \pm 5$ & $-65 \pm 5$ \\
\hline Absolute $\Delta \Phi(\mathrm{meV})$ & $-320 \pm 70$ & $-140 \pm 70$ & $-220 \pm 70$ & $-200 \pm 70$ & $-270 \pm 70$ \\
\hline
\end{tabular}


now on called "step 1" and "step 2"), and three different, electronically active GBs in CIGSe (from now on called "GB1", "GB2," and "GB3"). KPFS line profiles of both topography and work function across the NPDs are shown in Fig. 1. In Table I an overview of various properties of the NPDs is provided.

\section{KPFM AT NANOSCALE POTENTIAL DISTRIBUTIONS}

\section{A. Distance dependence}

In Fig. 2 details of a KPFM measurement at step 1 are shown. Figures 2(a) and 2(b) display line profiles of the topography and work function across the step respectively, across the step, taken at a tip-sample distance $z_{\min }=2.7 \mathrm{~nm}$. The corresponding KPFM images are shown as insets. While Fig. 2(a) indicates a step height of about $4 \mathrm{~nm}$, Fig. 2(b) displays a NPD at the step, with a minimal work function of $5.31 \mathrm{eV}$ directly at the center of the NPD and an average work function of $5.40 \mathrm{eV}$ in the background. Figure 2(c) shows
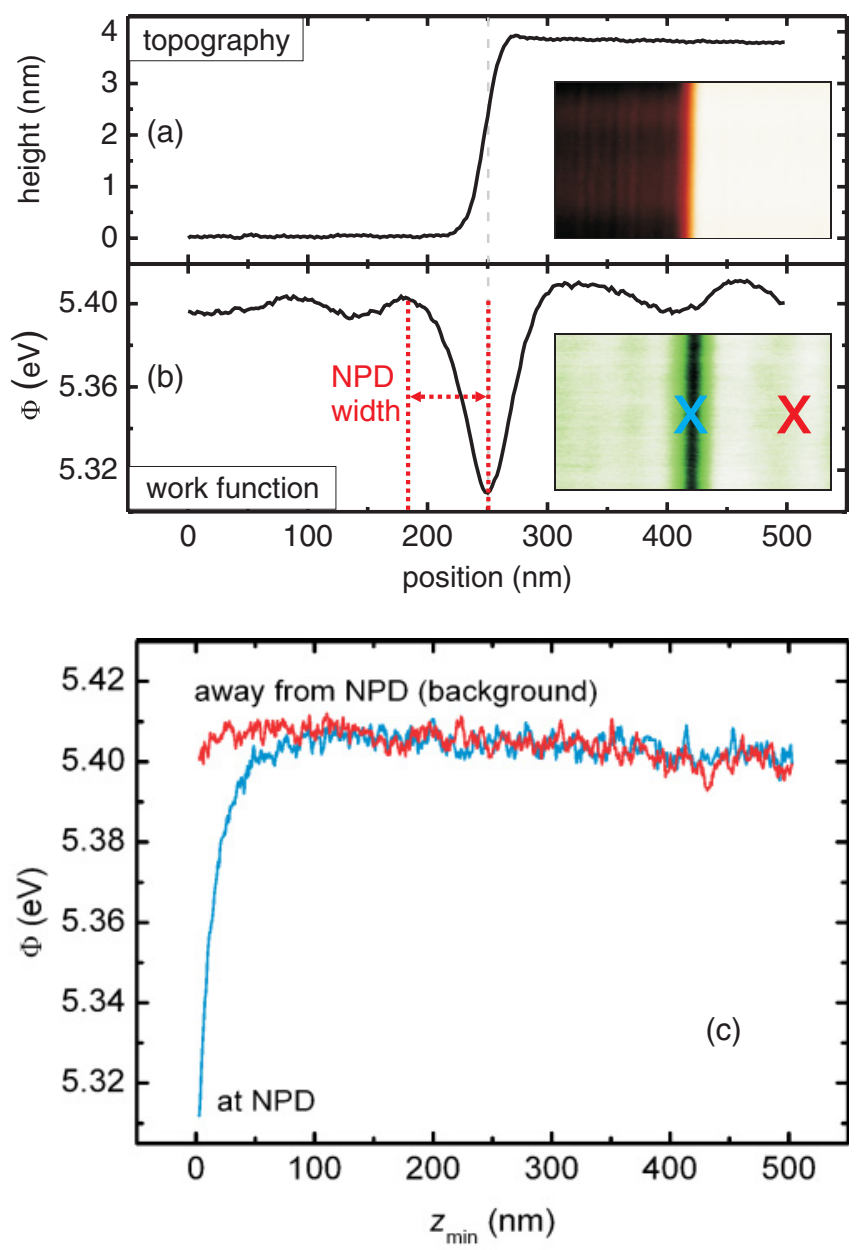

FIG. 2. (Color online) KPFM line profiles (ten lines averaged) of (a) topography and (b) work function $(\Phi)$ behavior across step 1, obtained at a tip-sample distance $z_{\min }=2.7 \mathrm{~nm} .500 \times 5 \mathrm{~nm}$ images of the corresponding surface area are shown as insets. (c) KPFS work function spectra at step 1, taken directly at the center of the NPD and in the background. The positions at which the spectra were taken are indicated in the insets of Fig. 2(b).

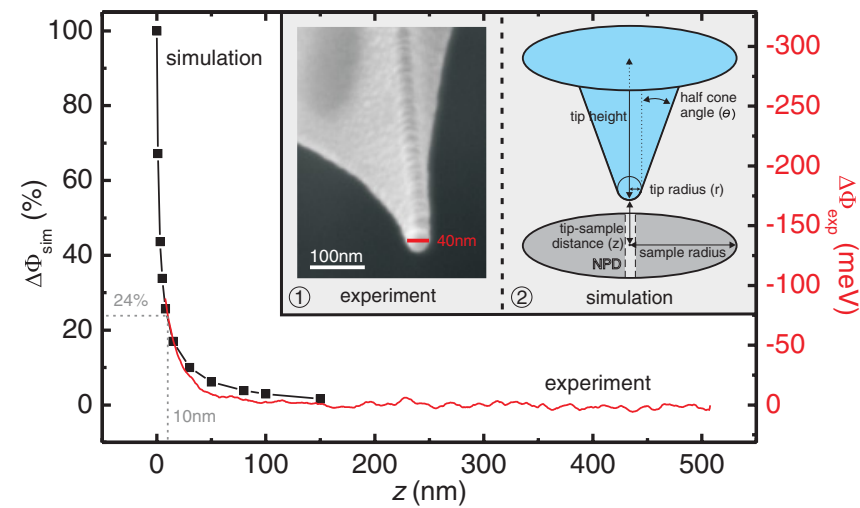

FIG. 3. (Color online) KPFS difference spectra of the NPD work function signal $(\Delta \Phi)$ of step 1, determined experimentally (line) and by simulations (squared line) (Ref. 38). Inset 1 shows an SEM image of the tip used for the experiment. Inset 2 shows the 3D tip geometry used for the simulation.

the KPFS work function spectra taken directly at the center of the NPD and at $\approx 150 \mathrm{~nm}$ distance from the NPD in the background. For the smallest tip-sample distance of $z_{\min }=2.7$ $\mathrm{nm}$ the spectrum at the NPD (blue line) reveals the same work function of $5.31 \mathrm{eV}$ as Fig. 2(b). With increasing tip-sample distance, the work function increases until it saturates at about $5.40 \mathrm{eV}$. In contrast, the background spectrum (red line) stays constant at around $5.40 \mathrm{eV}$, nearly independent of the tip-sample distance. This value is also consistent with Fig. 2(b). These findings indicate that the distance dependence of $\Phi$ above the NPD is caused only by averaging between the work function values of NPD and background.

In Fig. 3 the difference spectrum obtained from the data of Fig. 2(c) is shown. In order to quantify the distance dependence, the experimental data were reproduced by FEM simulations. (Note that here the experimental data are plotted versus the effective tip-sample distance $z$, accounting for the cantilever oscillation to allow comparison to the FEM simulations.) In the FEM simulations the absolute potential variation at the NPD is an input parameter. Consequently, it is possible to extrapolate the absolute work function variation of the experiment by fitting the simulation to the experimental data.

The simulation's input data for the tip geometry were obtained from scanning electron microscopy (SEM) images of the tip used in the experiment (see inset 1 in Fig. 3). The NPD width of the potential dip was obtained from the experimental data in Fig. 2(b). This is justified as it was previously shown that the width of a NPD is hardly affected by the averaging effect. $^{28}$ The topography step of about $4 \mathrm{~nm}$ was neglected in the simulation, as prior simulations did not show any impact of small steplike topographies on KPFM measurements. ${ }^{34}$

In order to fit the simulation to the experimental data, a scaling factor $s$ between the simulation's $y$ axis $\left[\Delta \Phi_{\text {sim }}(\%)\right]$ and the experimental $y$ axis $\left[\Delta \Phi_{\exp }(\mathrm{meV})\right]$ was used (see Fig. 3), which is defined via $\left[\Delta \Phi_{\exp }(\mathrm{meV})\right]=s\left[\Delta \Phi_{\operatorname{sim}}(\%)\right]$. A least-squares fit was applied to determine the optimum value of $s$. Thereby, the absolute value of the work function variation of the NPD at step 1 could be determined as $320 \pm 70 \mathrm{meV}$, which corresponds to an absolute work function of $\approx 5.08 \mathrm{eV}$ at 
the step edge (see Fig. 2). The inaccuracy in the determination of the absolute work function variation was obtained via error propagation from the inaccuracy of the experimental and simulation data. ${ }^{39}$

A strong distance dependence of the work function signal can be noticed from Fig. 3. At a tip-sample distance of 10 $\mathrm{nm}$, which is a typical working distance for cantilever-based KPFM imaging, only about $24 \%$ of the full NPD signal is detectable. Furthermore, the distance dependence curve displays a steep slope in the working regime of KPFM up to $\approx 30 \mathrm{~nm}$. This illustrates that work function signals of NPDs detected by KPFM are very sensitive with respect to the tip-sample distance.

\section{B. The impact of electronic inhomogeneities on KPFS experiments}

The KPFS work function spectra displayed in Fig. 2(c) show a nearly ideal behavior with increasing tip-sample distance. For small tip-sample distances the spectrum at the NPD yields the same work function value of the NPD as does the KPFM measurement in Fig. 2(b), while for larger tip-sample distances the work function increases until it saturates at the value of the constant, distance-independent, background spectrum.

Nevertheless, one cannot generally expect such ideal experimental spectra. Figures 4(a) and 4(b) show KPFS work function spectra taken at the NPDs of GB1, GB2, and GB3, and the corresponding background spectra. Additionally, spectra taken at two GaAs surface steps, step 1 (see Fig. 2) and step 2, are shown. All spectra were shifted such that the relative work function signal $\left(\Phi_{\text {rel }}\right)$ for large tip-sample distances is at 0 .

It is apparent that the KPFS spectra of the background of GB1-GB3 and step 2 deviate from the nearly constant behavior of step 1 for tip-sample distances up to $\approx 150 \mathrm{~nm}$. The corresponding spectra at the NPD also show an unexpected behavior with tip-sample distance, in particular in the cases of GB1 and step 2. While a decrease of $\Phi_{\text {rel }}$ is expected with decreasing tip-sample distance, due to the lower work function of the NPDs, both spectra yield an increase of $\Phi_{\text {rel }}$ for small tip-sample distances. The similarity of the topography of the two GaAs(110) surface steps indicates that these deviations in the spectra are not associated with surface topography. We propose that the deviations are due to an inhomogeneous work function distribution of the tip; a different tip was used for the measurements at step 1 from the one used for those at GB1-GB3 and step 2. The inset of Fig. 5(a) shows an SEM image of the tip used for the measurements at GB1-GB3 and step 2, clearly presenting a contamination at its apex. At different tip-sample distances different areas of the tip contribute to the work function signal; therefore, this inhomogeneity likely caused the deviations in the spectra. In principle, inhomogeneities of the sample background can also cause deviations in the spectra, as the averaged surface area increases with increasing tip-sample distance. However, such inhomogeneities should mainly have an influence for small tip-sample distances $\left(z_{\min }<50 \mathrm{~nm}\right)$, since they are averaged out for larger tip-sample distances. Additionally, significant background inhomogeneities were not observed in the vincinity of the studied NPDs (see Fig. 1). Consequently,
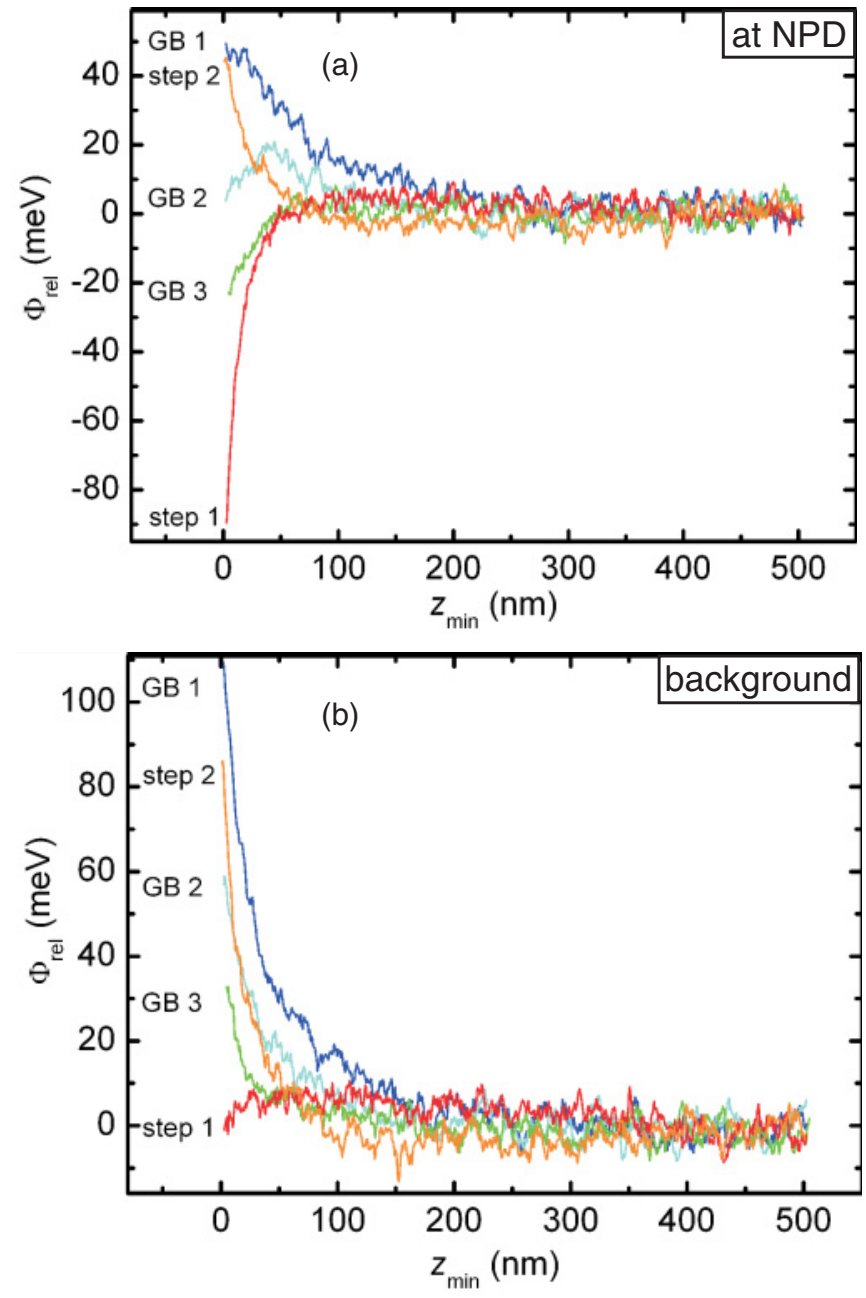

FIG. 4. (Color online) KPFS work function spectra taken at various NPDs of CIGSe grain boundaries and GaAs surface steps (see Table I). Spectra taken (a) at the center of the NPDs and (b) in the corresponding backgrounds. All work function spectra were shifted in such a way that the relative work function $\left(\Phi_{\text {rel }}\right)$ is at 0 for large tip-sample distances.

we can exclude background inhomogeneities as the source of the deviations in Figs. 4(a) and 4(b).

To avoid any influence of electronic inhomogeneities on the KPFS experiments it is necessary to subtract the background spectra from the corresponding spectra at the center of the NPDs. By means of the subtraction the deviations in the spectra are canceled out. This can be seen in Fig. 5(a), showing the resulting difference spectra from the spectra shown in Fig. 4. They yield a similar distance dependence, independent of the cantilever used.

\section{Influence of surface topography}

In order to investigate the influence of surface topography on the averaging effect, the distance dependence at the NPDs of the GaAs step edges and the CIGSe GBs is compared. In contrast to the $\mathrm{GaAs}(110)$ step edges, which show a negligible surface topography, the GBs in CIGSe show a significant valleylike topography (see Fig. 1). An influence of this topography on the averaging effect could be possible, 

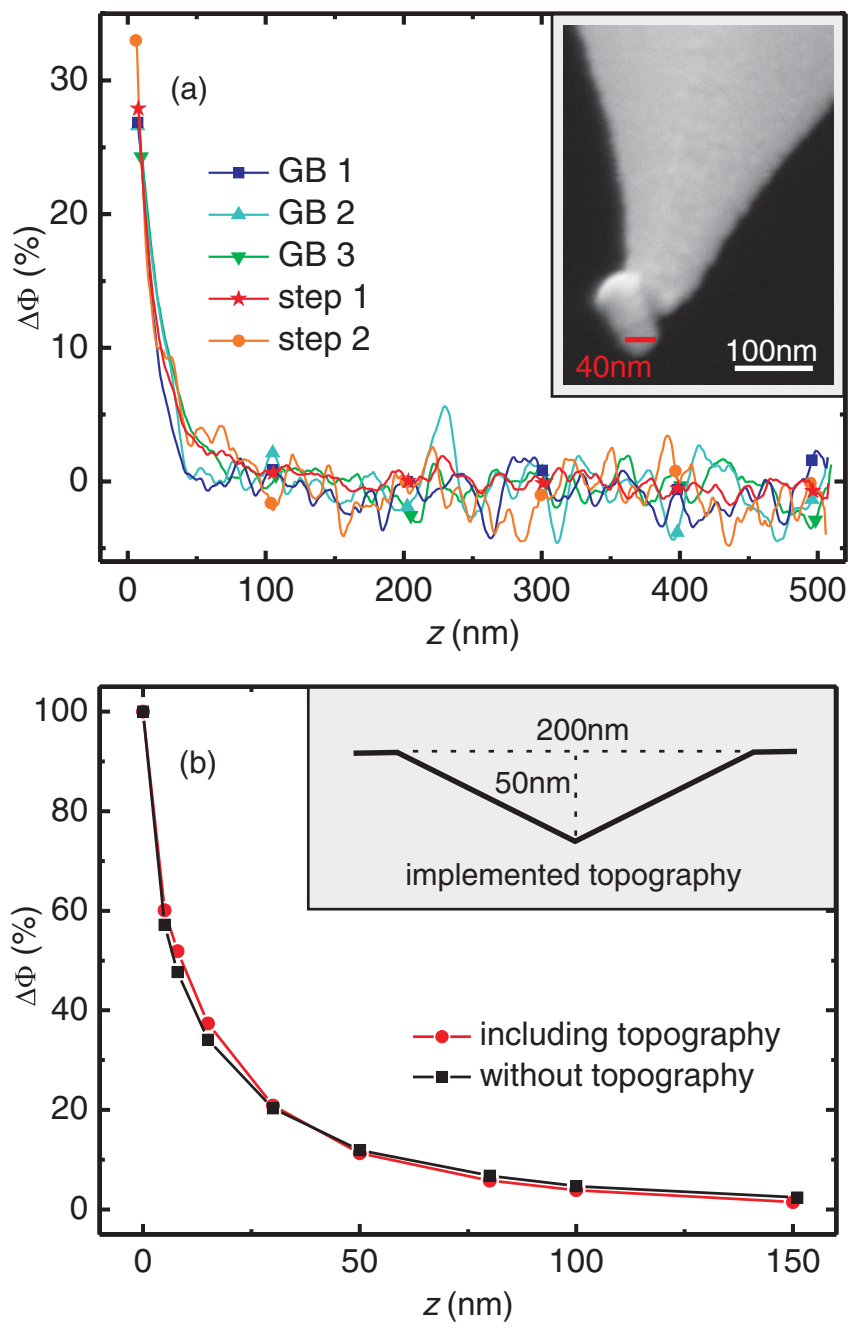

FIG. 5. (Color online) (a) Experimental KPFS difference spectra of the NPD work function signal $(\Delta \Phi)$ taken at GB1-GB3, step 1, and step 2. All spectra were normalized to the absolute NPD signal percentage from Fig. 3 at the smallest common tip-sample distance $z=9.7 \mathrm{~nm}$. The inset shows an SEM image of the tip used for the KPFS experiments at GB1-GB3 and step 2. (b) Simulated (Ref. 40) KPFS difference spectra of the NPD work function signal $(\Delta \Phi)$ with and without the surface topography displayed in the inset.

since a stronger interaction between the tip and the sides of the valley might reduce or enhance the averaging effect.

Figure 5(a) shows the KPFS difference spectra of the NPD work function signal for the three different GBs (GB1, GB2, and GB3) and the two differently doped $p$-type $\operatorname{GaAs}(110)$ surface steps (step 1 and step 2). All spectra were normalized to the absolute NPD signal percentage from Fig. 3 at the smallest common tip-sample distance $z=9.7 \mathrm{~nm}$. Despite the strongly different surface topographies of GB1-GB3 and the much smaller topography of the surface steps, no influence of the topography on the KPFS difference spectra can be seen. Especially for smaller tip-sample distances up to $30 \mathrm{~nm}$, which is the relevant working range for KPFM, the spectra are congruent. The rather large scattering of the spectra GB1-GB3 and step 2, compared to step 1, especially for large tip-sample distances, is mainly caused by a reduced signal-to-noise ratio for these spectra. Because of the similar distance dependence of the spectra, it is possible to estimate the magnitude of the averaging effect from Fig. 5. The obtained absolute potential variations at the five NPDs are given in Table I.

The negligible influence of surface topography on the averaging effect is also confirmed by simulations, as can be seen in Fig. 5(b). The simulated KPFS difference spectra of the NPD work function signal are identical within the experimental noise level, with and without the surface topography indicated in the inset.

\section{DISCUSSION}

Our results reveal a significant averaging effect of the work function signal of NPDs in KPFM, which basically prohibits any direct quantitative comparison of results among different experiments or to theory. In this paper we provide a guideline for how to overcome this problem and to obtain absolute work function values from KPFM measurements at NPDs by additionally consulting FEM simulations.

As demonstrated in Fig. 5(a), it is reasonable to transfer our results from Fig. 3 to KPFM measurements at different NPDs, if tips with a similar geometry were used for the experiments. It is not mandatory to exactly determine the radius of the used tip. As shown in Fig. 6, the tip radius has only a minor influence on the distance dependence, considering the typical experimental noise level. To account for different oscillation amplitudes, one can revert to effective tip-sample distances, as provided in Ref. 34. Additionally, the observed distance dependence appears to be fairly robust with respect to the NPD width. No variation in the distance dependence between 20 and $65 \mathrm{~nm}$ NPD width could be resolved experimentally [see Fig. 5(a)]. In principle, however, FEM simulations have shown that width of a NPD should affect the averaging effect. ${ }^{34}$

The transfer of the results from Fig. 3 to KPFM measurements at different NPDs is additionally simplified by the finding that surface topography has no significant influence on the averaging effect.

Finally, we would like to point out that the presented experimental as well as simulation results are based on the physics of

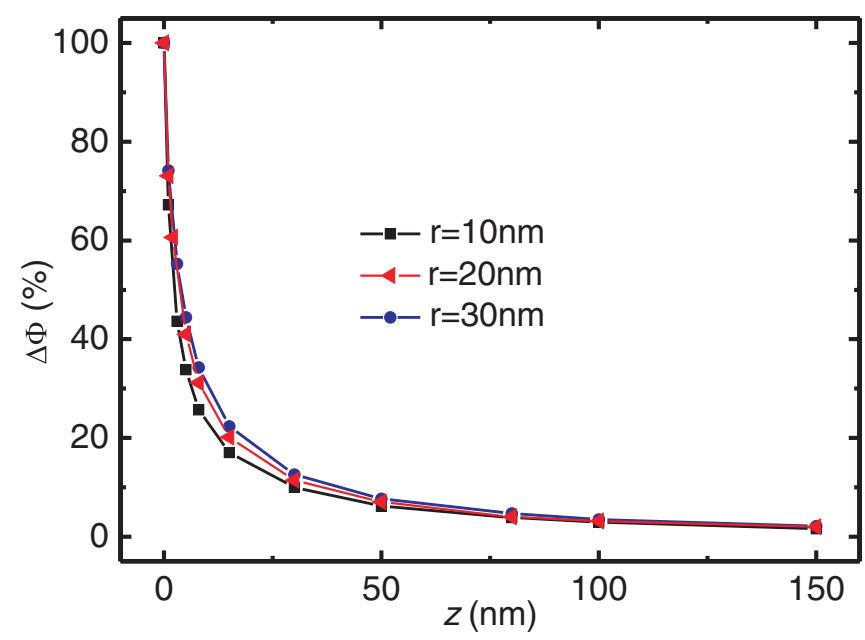

FIG. 6. (Color online) Simulated (Ref. 41) KPFS difference spectra of the NPD work function signal $(\Delta \Phi)$ for different radii $r$ of the tip apex. 
the work function, a macroscopic materials property. We can therefore not claim applicability of our method to atomic-scale CPD variations, ${ }^{16-18}$ since these presumably have a different physical origin.

\section{CONCLUSIONS}

In summary, we have demonstrated a strong distance dependence of the averaging effect of NPD work function signals in KPFM measurements, stressing its importance in calibration of the tip-sample distance. By combining KPFS in experiment and simulation we were able to quantify this averaging effect, which enables quantitative comparison among different KPFM experiments and to theory. Our investigations did not reveal any influence of surface topography on the averaging effect.

\section{ACKNOWLEDGMENT}

The authors gratefully acknowledge financial support from the Bundesministerium für Umwelt, Naturschutz und Reaktorsicherheit (BMU) under Contract No. 0327559H. *robert.baier@helmholtz-berlin.de

${ }^{1}$ G. Binnig, C. F. Quate, and C. Gerber, Phys. Rev. Lett. 56, 930 (1986).

${ }^{2}$ F. J. Giessibl, Phys. Rev. B 56, 16010 (1997).

${ }^{3}$ J. M. R. Weaver and H. K. Wickramasinghe, J. Vac. Sci. Technol. B 9, 1562 (1991).

${ }^{4} \mathrm{M}$. Nonnenmacher, M. Oboyle, and H. Wickenramasinghe, Appl. Phys. Lett. 58, 2921 (1991).

${ }^{5}$ S. Sadewasser, T. Glatzel, M. Rusu, A. Jäger-Waldau, and M. C. Lux-Steiner, Appl. Phys. Lett. 80, 2979 (2002).

${ }^{6}$ L. Gross, F. Mohn, P. Liljeroth, J. Repp, F. J. Giessibl, and G. Meyer, Science 324, 1428 (2009).

${ }^{7}$ Y. Rosenwaks, R. Shikler, T. Glatzel, and S. Sadewasser, Phys. Rev. B 70, 085320 (2004).

${ }^{8}$ M. Hafemeister, S. Siebentritt, J. Albert, M. C. Lux-Steiner, and S. Sadewasser, Phys. Rev. Lett. 104, 196602 (2010).

${ }^{9}$ Z. Zhang, X. Tang, U. Lemmer, W. Witte, O. Kiowski, M. Powalla, and H. Hölscher, Appl. Phys. Lett. 99, 042111 (2011).

${ }^{10}$ C. Loppacher, U. Zerweck, and L. Eng, Nanotechnology 15, S9 (2004).

${ }^{11}$ C. Barth and C. R. Henry, Phys. Rev. Lett. 98, 136804 (2007).

${ }^{12}$ F. Bocquet, L. Nony, C. Loppacher, and T. Glatzel, Phys. Rev. B 78, 035410 (2008).

${ }^{13}$ H. Hoppe, T. Glatzel, M. Niggemann, A. Hinsch, M. Lux-Steiner, and N. Sariciftci, Nano Lett. 5, 269 (2005).

${ }^{14}$ O. Tal, Y. Rosenwaks, Y. Preezant, N. Tessler, C. K. Chan, and A. Kahn, Phys. Rev. Lett. 95, 256405 (2005).

${ }^{15}$ S. Sadewasser and M. C. Lux-Steiner, J. Vac. Sci. Technol. B 28, 1071 (2010)

${ }^{16}$ S. Kitamura, K. Suzuki, M. Iwatsuki, and C. B. Mooney, Appl. Surf. Sci. 157, 222 (2000).

${ }^{17}$ G. H. Enevoldsen, T. Glatzel, M. C. Christensen, J. V. Lauritsen, and F. Besenbacher, Phys. Rev. Lett. 100, 236104 (2008).

${ }^{18}$ S. Sadewasser, P. Jelinek, C.-K. Fang, O. Custance, Y. Yamada, Y. Sugimoto, M. Abe, and S. Morita, Phys. Rev. Lett. 103, 266103 (2009).

${ }^{19}$ L. Nony, A. S. Foster, F. Bocquet, and C. Loppacher, Phys. Rev. Lett. 103, 036802 (2009).

${ }^{20}$ A. Masago, M. Tsukada, and M. Shimizu, Phys. Rev. B 82, 195433 (2010).

${ }^{21}$ T. Hochwitz, A. K. Henning, C. Levey, C. Daghlian, and J. Slinkman, J. Vac. Sci. Technol. B 14, 457 (1996).
${ }^{22}$ J. Colchero, A. Gil, and A. M. Baró, Phys. Rev. B 64, 245403 (2001).

${ }^{23}$ G. Elias, T. Glatzel, E. Meyer, A. Schwarzman, A. Boag, and Y. Rosenwaks, Beilstein J. Nanotechnol. 2, 252 (2011).

${ }^{24}$ H. O. Jacobs, P. Leuchtmann, O. J. Homan, and A. Stemmer, J. Appl. Phys. 84, 1168 (1998).

${ }^{25}$ S. Sadewasser, T. Glatzel, R. Shikler, Y. Rosenwaks, and M. LuxSteiner, Appl. Surf. Sci. 210, 32 (2003).

${ }^{26}$ E. Palleau, L. Ressier, L. Borowik, and T. Mélin, Nanotechnology 21, 225706 (2010).

${ }^{27}$ S. Belaidi, F. Lebon, P. Girard, G. Leveque, and S. Pagano, Appl. Phys. A 66, 239 (1998).

${ }^{28}$ C. Leendertz, F. Streicher, M. C. Lux-Steiner, and S. Sadewasser, Appl. Phys. Lett. 89, 113120 (2006).

${ }^{29}$ U. Zerweck, C. Loppacher, T. Otto, S. Grafström, and L. M. Eng, Phys. Rev. B 71, 125424 (2005).

${ }^{30}$ C. Sommerhalter, T. Matthes, T. Glatzel, A. Jäger-Waldau, and M. C. Lux-Steiner, Appl. Phys. Lett. 75, 286 (1999).

${ }^{31}$ C. Sommerhalter, T. Glatzel, T. Matthes, A. Jäger-Waldau, and M. Lux-Steiner, Appl. Surf. Sci. 157, 263 (2000).

${ }^{32}$ EFM-PPP cantilevers from Nanoworld were used for all experiments.

${ }^{33}$ C. Leendertz, Diplomarbeit, Freie Universität Berlin, 2006.

${ }^{34}$ S. Sadewasser, C. Leendertz, F. Streicher, and M. C. Lux-Steiner, Nanotechnology 20, 505503 (2009).

${ }^{35}$ This procedure causes an inaccuracy in $z$ for large tip-sample distance, as the effective tip-sample distance $z$ depends on $z_{\min }$. However, considering the course of our experimental KPFS spectra (constant for large $z$ ), no effect on the experimental results is expected.

${ }^{36}$ J. Seto, J. Appl. Phys. 46, 5247 (1975).

${ }^{37}$ S. Sadewasser, T. Glatzel, S. Schuler, S. Nishiwaki, R. Kaigawa, and M. C. Lux-Steiner, Thin Solid Films 431-432, 257 (2003).

${ }^{38}$ Simulation parameters: tip height $13 \mu \mathrm{m}$, sample radius $25 \mu \mathrm{m}$, $\theta=30^{\circ}, r=10 \mathrm{~nm}$, SCR $65 \mathrm{~nm}$.

${ }^{39} \mathrm{An}$ inaccuracy of $4 \mathrm{meV}$ was assumed for the experimental data based on the noise level, and for the simulation data the inaccuracy was estimated as $2 \%$ based on Fig. 6.

${ }^{40}$ Simulation parameters: tip height $13 \mu \mathrm{m}$, sample radius $25 \mu \mathrm{m}$, $\theta=10^{\circ}, r=30 \mathrm{~nm}$, SCR $65 \mathrm{~nm}$.

${ }^{41}$ Simulation parameters: tip height $13 \mu \mathrm{m}$, sample radius $25 \mu \mathrm{m}$, $\theta=30^{\circ}, r$ various, SCR $65 \mathrm{~nm}$. 